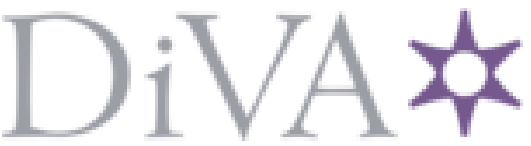

http://www.diva-portal.org

Postprint

This is the accepted version of a paper presented at 2012 International Conference on Optical Engineering (ICOE).

Citation for the original published paper:

Khodashenas, N S., Shahini, A., Sayyad Khodashenas, P., Maleki, H., Abbasian, K. (2012)

Effect of charge carriers and excitons mobility on transport in an organic solar cell In:

N.B. When citing this work, cite the original published paper.

Permanent link to this version:

http://urn.kb.se/resolve?urn=urn:nbn:se:kth:diva-282946 


\section{Effect of Charge Carriers and Excitons Mobility on Transport in an Organic Solar Cell}

\author{
A. Shahini \\ School of engineering \\ emerging technologies \\ University of Tabriz \\ Tabriz, Iran \\ ali.shahini.62@gmail.com
}

\author{
P. S. Khodashenas \\ CCABA Center \\ Universitat Politècnica \\ de Catalunya \\ Barcelona, Spain
}

\author{
H. F. Maleki \\ Islamic azad \\ University \\ Malekan branch \\ Malekan, Iran
}

\author{
N. S. Khodashenas \\ Islamic azad University \\ Lahijan branch \\ Lahijan, Iran
}

\author{
K. Abbasian \\ The Photonics and \\ Nanocrystal \\ Research Lab \\ University of Tabriz \\ Tabriz, Iran
}

\begin{abstract}
An organic solar cell model is developed that consists of both excitonic and classical bipolar aspects of solar cells. In order to be compatible with the principle of detailed balance theory we have imported the photon recycling term in our equations. As a result we connected the Shockley-Queisser (SQ) theory to the classical diode theory. This model for excitonic and classical bipolar solar cells can describes the combined transport and interaction of electrons, holes and excitons. For high mobilities our model reproduces the Shockley Queisser efficiency limit. We show how varying the respective mobilities of the different species changes the operation mode of the solar cell path between excitonic and bipolar.
\end{abstract}

Keywords-component; mobility; photon recycling; short circuit current density; exciton

\section{INTRODUCTION}

The introduction of organic materials as photovoltaic absorbers in organic [1-4] or dye-sensitized solar cells has raised questions whether or not these new types of devices can be described with the help of theories that initially have been developed for inorganic solid-state type devices usually provided with a pn-homo- or heterojuncion. However, so far, these so-called second generation [5] thin-film solar cells have not yet lived up to expectations. They all fall short of achieving the efficiencies reached with conventional first-generation silicon solar cells. Within these years, our understanding of this device, its working principles, and its processing has obviously achieved a high degree of maturity. Recently, it has been proposed $[6,7]$ to distinguish between two different concepts of solar cells, namely, the classical inorganic solar cells and the excitonic solar cells usually made from organic absorber materials. However, the overall functionality in all solar cells, namely, the generation of electrical power from solar light, is identical, and there should be a common theory that is valid for all devices on a certain level of abstraction from physical details. The present paper proposes a model that allows us to describe virtually all single-absorber solar cells. For the bulk of the absorber, we use a set of differential equations for excitons, electrons, and holes that is coupled by the dissociation of excitons into an electron/hole pair and the recombination of this pair into an exciton. This approach is similar as in $[8,9]$. At the surfaces of the absorber, we allow for cross-dissociation of the exciton into an electron in the absorber and a hole in the contact and vice versa.

The paper is organized as follows. We start with a discussion of our model and show how the choice of the boundary conditions allows us to simulate p-i-n-type solar cells. The discussion of the results starts with simulation of current-voltage curve for different exciton mobilities, and then we discuss the current-voltage characteristics of $p$-i-n-type solar cells for different charge carrier mobilities.

\section{MODEL}

To be compatible with the maximum quantum efficiency of Shockley and Queisser and also connect this SQ theory with classical diode theory, the absorption of photons emitted by the device itself has to be taken into account. This effect of optical coupling in the absorber is usually referred to as photon recycling [10]. In order to drive the current densities for electrons, holes and exitons, we have to calculate first the carrier and exciton concentrations. To calculate the carrier and exciton concentrations as a function of depth in the absorber, four differential equations have to be solved, namely, poisson equation $\Delta \varphi=-\rho / \varepsilon$, relating the electrical potential $\varphi$ to the space charge $\rho$ and the dielectric constant $\varepsilon$, and three continuity equations for electrons, holes and excitons .

$$
\begin{aligned}
& D_{\chi} \frac{d^{2} \chi}{d x^{2}}=\frac{\chi}{\tau_{D}}+\frac{\chi}{\tau_{r}}-R_{\chi} n p-g_{\chi}, \\
& D_{n} \frac{d^{2} n}{d x^{2}}+F \mu_{n} \frac{d n}{d x}=-\frac{\chi}{\tau_{D}}+R_{\chi} n p \\
& D_{p} \frac{d^{2} p}{d x^{2}}-F \mu_{p} \frac{d p}{d x}=-\frac{\chi}{\tau_{D}}+R_{\chi} n p
\end{aligned}
$$

Here, $g_{\chi}$ is the optical generation rate for excitons, $R_{\chi}$ is the recombination rate of free carriers leading to the creation of excitons, $\tau_{D}$ is the bulk dissociation lifetime of the excitons, $\tau_{r}$ is the radiative lifetime of the excitons, $F$ is the electric field, $\mu_{\chi / n / p}$ are the mobilities, and $D_{\chi / n / p}=\mu_{\chi / n / p} k T / q$ are the diffusion constants according to Einstein's equation, where $k T$ is the thermal energy and $q$ is the elementary charge. In the nonequilibrium situation, the interface dissociation and 
recombination process defines the boundary condition for these equations. The exciton current density at the cordinate $x=0$ is defined by $j_{\chi}=S_{\chi n} n(0)-H_{n} n_{j} p(0)$. Similarly, $j_{n}$ is defined by $j_{n}=S_{n} n(0)-S_{n}^{*} n_{j}$ such that $j_{n}$ corresponds to the net number of free electrons collected by the junction at $x=0$ whereas $j_{\chi}$ counts the number of excitons that are separated into electrons (in the junction) and holes (in the bulk) at that interface (Fig. 1). Because of our assumption that excitons only dissociate at the light exposed surface of the absorber $(x=0)$, the hole current density (analyzed at $x=d$ ) corresponds to the $\operatorname{sum} j_{p}=j_{n}+j_{\chi}$. Here $H_{n}$ is cross recombination rate of electrons in the junction with holes in the bulk of the absorber, $S_{\chi_{n}}$ is dissociation velocity of excitons at the electron contact, and $S_{n}, S_{n}^{*}$ have the dimension of a (collection or injection) velocity.
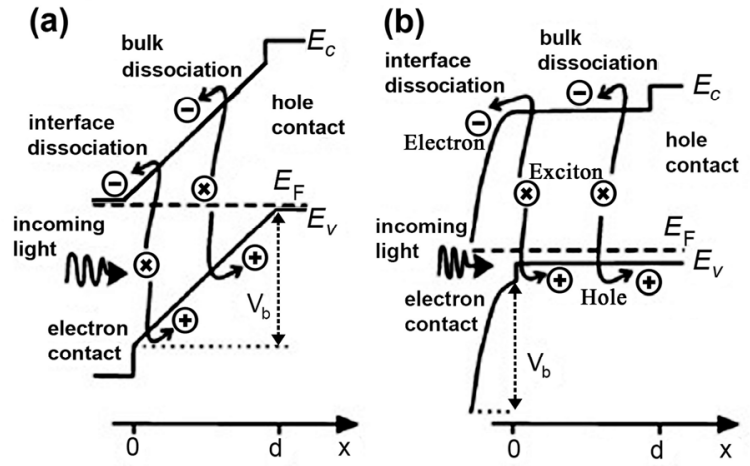

Figure 1. Scheme of the (a) $p$ - $i$ - $n$-junction device and the (b) $p n$-junction device. For both types the photogenerated exciton can be either split in the bulk (bipolar case) or at the junction (excitonic case) of the device.

\section{RESULTS}

In order to focus on transport mechanism in excitonic and bipolar solar cells, the following computations are restricted to the short circuit situation of pin-type solar cells. Also, for all simulations we have used a set of constant parameters, which are shown in table 1 . The absorption coefficient of $\mathrm{ZnPc}$ as a typical organic absorber material (taken from Fig. 4 in [11]), an optical generation profile, and a photon recycling scheme calculated according to above that both result from a Lambertian cell surface.

Table 1. Constant parameters for a p-i-n type solar cell. $V_{b i}$ is equilibrium biltin potential inside the absorber and $\chi_{0}$ is the equilibrium concenteration of excitons

\begin{tabular}{|c|c|c|c|c|}
\hline \multicolumn{5}{|c|}{ Constant parameters } \\
\hline $\begin{array}{c}\text { Thickness } \\
(\boldsymbol{d})\end{array}$ & $\begin{array}{c}\text { Intrinsic } \\
\text { carrier } \\
\text { concentration } \\
\left(\boldsymbol{n}_{i}\right)\end{array}$ & $\begin{array}{c}\text { Equilibrium } \\
\text { bilt-in } \\
\text { potential }\left(V_{b i}\right)\end{array}$ & $\begin{array}{c}\text { Recombination } \\
\text { lifetime }\left(\boldsymbol{\tau}_{r}\right)\end{array}$ & $\begin{array}{c}\text { Equilibrium } \\
\text { exciton } \\
\text { concentration } \\
\left(\chi_{0}\right)\end{array}$ \\
\hline $300 \mathrm{~nm}$ & $10^{3} \mathrm{~cm}^{-3}$ & $1.3 \mathrm{~V}$ & $200 \mu \mathrm{s}$ & $4.4 \times 10^{-3} \mathrm{~cm}^{-3}$ \\
\hline
\end{tabular}

Fig. 2 shows simulated current/voltage curves for a solar cell with a fixed electron and hole mobility $\mu_{n / p}=10^{3} \mathrm{~cm}^{2} \mathrm{v}^{-1} \mathrm{~s}^{-1}$ and a fixed dissociation lifetime $\tau_{\mathrm{D}}=10^{-4} \mathrm{~s}$. This long dissociation lifetime corresponds to a situation that is excitonic for high exciton mobilities .The dashed curve corresponding to the high exciton mobility equals the SQ limit, Such that, every absorbed photon contributes one elementary charge to the short circuit current. For lower mobilities, the decreased collection of excitons leads to the situation where the quantum efficiency becomes smaller than the absorptance and $J_{\text {sc }}$ decreases. The open circuit voltage as well as the fill factor remains almost unaffected by the change in exciton mobility.

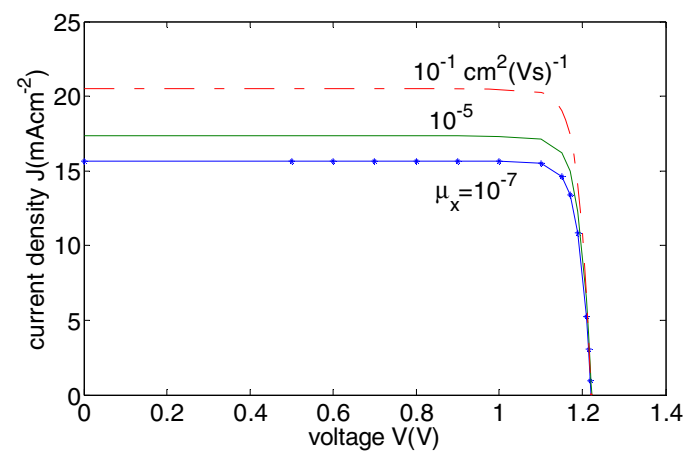

Figure 2. Simulation of current-voltage curves for a solar cell with a fixed electron and hole mobility and a fixed dissociation lifetime.

A more detailed analysis of the short circuit current density $J_{\text {sc }}$ and the contributions to the current density $J_{n}$ and $J_{\chi}$ from electrons and excitons is depicted in Fig. 3 as a function of exciton mobility. Obviously, a decreasing $\mu_{\chi}$ hampers the current collection by excitonic diffusion leading to a decrease of $J_{\chi}$ starting at approximately $\mu_{\chi} \approx 2 \times 10^{-3} \mathrm{~cm}^{2} \mathrm{~V}^{-1} \mathrm{~s}^{-1}$. As soon as $J_{\chi}$ starts to decline, we observe an increase of $J_{n}$. Obviously, part of the excitonic current is taken over by the electrons and the device changes their character from excitonic to bipolar. However, the overall currents $J_{\mathrm{sc}}=J_{\mathrm{p}}=J_{n}+J_{\chi}$ are smaller in the bipolar than in the excitonic limit. This is because of the fact that the dissociation lifetime $\tau_{\mathrm{D}}=100 \mu \mathrm{s}$ is chosen comparable to the radiative recombination lifetime $\tau_{\mathrm{r}}=200 \mu \mathrm{s}$. Therefore, a part of the photogenerated excitons just recombines before they dissociate into electron/hole pairs that are quickly separated in both devices due to the assumed high mobilities $\mu_{n}=\mu_{p}=10^{3} \mathrm{~cm}^{2} \mathrm{~V}^{-1} \mathrm{~s}^{-1}$. Despite of the fact that radiative recombination is the only loss mechanism assumed in the present calculations, device did not achieve full carrier collection. Consequently, device falls short of the SQ-limit if the exciton mobility is below a critical value and if the dissociation lifetime is longer or comparable with the recombination lifetime.

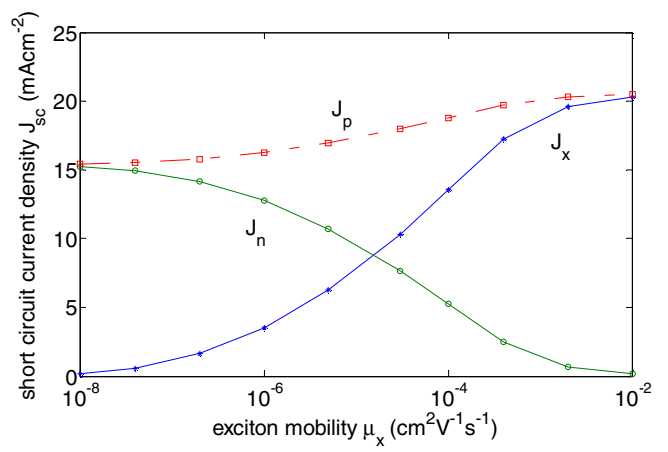

Figure 3. Exciton, electron, and hole contribution $J_{\chi}, J_{n}$, and $J_{\mathrm{p}}$ to the short circuit current density for different values of the exciton mobility with a fixed dissociation lifetime corresponding to the excitonic limit.

Fig. 4 shows a simulation of current-voltage curve for different carrier mobilities. Here, we have chosen very short 
dissociation lifetime $\left(\tau_{\mathrm{D}}=1 \mathrm{~ns}\right)$. At high mobilities of free carriers $\mu_{n, p} \geq 10^{3} \mathrm{~cm}^{2} \mathrm{~V}^{-1} \mathrm{~s}^{-1}$, this short dissociation lifetime corresponds to a bipolar photocurrent under short circuit conditions. Here, we vary the carrier mobility $\mu_{n, p}=10^{0}, 10^{1}$, $10^{3} \mathrm{~cm}^{2} \mathrm{~V}^{-1} \mathrm{~s}^{-1}$. The decreasing carrier mobility leads to a decay in fill factor and in short circuit current density. However, the fill factor loss is by far more dominant than that in $J_{s c}$. This is because for low charge carrier mobilities in p-in-type solar cells, the effect of the built-in field becomes decisive. Since the charge carriers; unlike the excitons, react on the decrease in the built-in field resulting by application of forward bias voltage $V$, the photocurrent decreases with increasing $V$. The voltage dependence of carrier collection reduces the fill factor. Therefore, the fill factor is strongly influenced by the carrier mobilities although the open circuit voltage is not dependent on $\mu_{n, p}$ in Fig. 4.

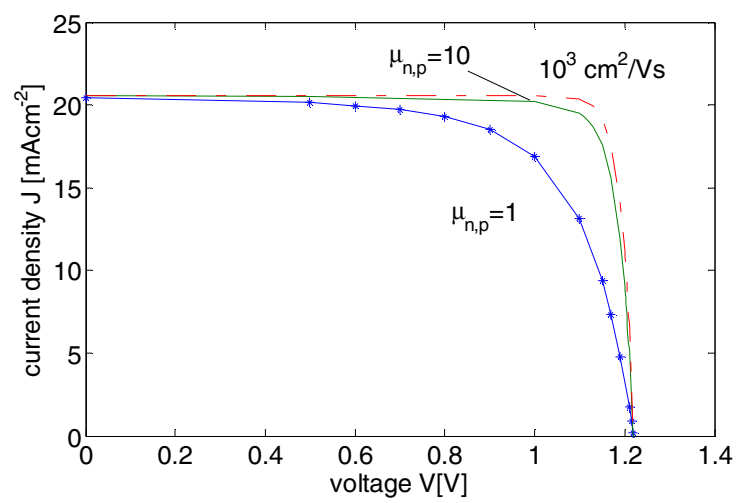

Figure 4. Simulation of current-voltage curves for a solar cell with a fixed exciton mobility $\mu_{\chi}=10^{-5} \mathrm{~cm}^{2} \mathrm{~V}^{-1} \mathrm{~s}^{-1}$ and a fixed dissociation lifetime $\tau_{\mathrm{D}}=10^{-10}$

Fig. 5 shows the influence of the electron mobility on the charge carrier collection properties of the pin solar cell. As expected, the electron contribution decreases with decreasing $\mu_{n}$. However, the exciton contribution increases at the same time and fully compensates the loss in $J_{n}$. Thus, the device changes its character from bipolar to excitonic without any losses in $J_{\mathrm{sc}}$. This example shows that for the present case of two competing transport channels, a decreasing mobility of one type of particle does not necessarily lead to short circuit currents below the SQ-limit.

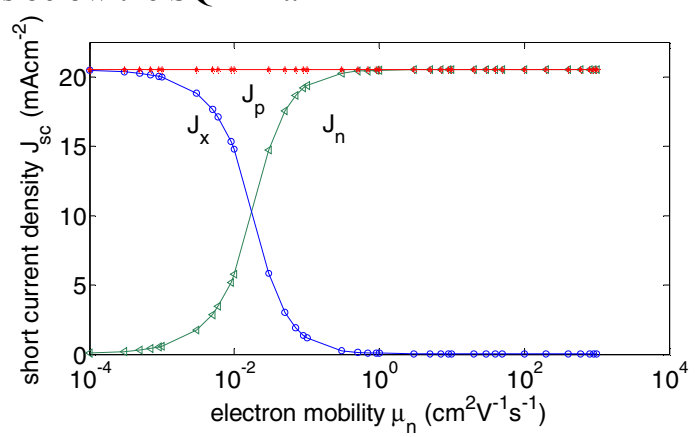

Figure 5. Exciton, electron, and hole currents $J_{\gamma}, J_{n}$, and $J_{p}$ for different values of the electron mobility with a fixed dissociation lifetime.

\section{CONCLUSIONS}

The present paper has introduced a model for solar cells that connects the Shockley-Queisser theory to the classical diode theory and includes transport and interaction of excitons, electrons, and holes. The model is designed to be compatible with the SQ efficiency limit. Also the model is able to describe a continuous transition between an excitonic and a bipolar operation mode of pin-as well as pn-type solar cells. In the excitonic limit, for exciton mobilities below a critical value our model did not achieve full carrier collection and, device falls short of the SQ-limit. In the bipolar limit, the dissociation of excitons takes place already in the bulk of the photovoltaic absorber and our model reproduce SQ maximum efficiency limit. Photovoltaic charge separation, in this limit, results with the help of the built-in field and/or by the selectivity of the contacts.

\section{ACKNOWLEDGMENT}

The Authors would like to thank M. Heidari, A. Soltanieh, H. Soofi and H. Nadali for fruitful discussions.

\section{REFERENCES}

[1] C.W. Tang, "Two-layer organic photovoltaic cell," Appl. Phys. Lett, vol. 48, pp. 183-185, 1986.

[2] H. Lu, B. Akgun and T. B. Russell, "Morphological characterization of a low-bandgap crystalline polymer: PCBM bulk heterojunction solar cells,” Adv. Eng. Mat, vol. 1, pp. 870-878, 2011.

[3] H. Park, J. A. Rowehl, K. K. Kim, V. Bulovic and J. Kong, "Doped graphene electrodes for organic solar cells," IOP. Science. Nanotechnology, vol. 21, No. 50, 2010.

[4] S. Bertho et al., "Effect of temperature on the morphological and photovoltaic stability of bulk heterojunction polymer: Fullerene solar cells," Sol. Energy. Mater. Sol. Cells, vol. 92, pp. 753-760, 2008.

[5] M. A. Green, "Third generation photovoltaics: solar cells for 2020 and beyond," Physica, vol. E 14, pp. 65-70, 2002.

[6] B. A. Gregg and M. C. Hanna, "Comparing organic to inorganic photovoltaic cells: Theory, experiment, and simulation," J. Appl. Phys, vol. 93, pp. 3605-3614, 2003.

[7] B. A. Gregg, "Excitonic solar cells," J. Phys. Chem, vol. B 107, pp. 4688-4698, 2003.

[8] R. Corkish, D.S.P. Chan and M. A. Green, "Excitons in silicon diodes and solar cells: A three-particle theory,"J. Appl. Phys, vol. 79, pp. 195$203,1996$.

[9] M. Burgelman and B. Minnaert, "Including excitons in semiconductor solar cell modelling," Thin. Solid. Films, vol. 511, pp. 214-218, 2006.

[10] J. Mattheis, J. H. Werner, and U. Rau, "Finite mobility effects on the radiative efficiency limit of $p n$-junction solar cells," Phys. Rev. B, vol. 77, pp. 085203-085215, 2008.

[11] J. Rostalski and D. Meissner, "Photocurrent spectroscopy for the investigation of charge carrier generation and transport mechanisms in organic p-n junction solar cells," Sol. Energy. Mater. Sol. Cells, vol. 63, pp. $37-47,2000$. 